\title{
ELECCIÓN DE CARRERA EN ESTUDIANTES DE NIVEL SOCIOECONÓMICO BAJO DE UNIVERSIDADES CHILENAS ALTAMENTE SELECTIVAS
}

\section{Introducción}

En las últimas décadas se ha cuadruplicado el número de jóvenes entre 18 y 24 años que acceden a la educación superior en Chile, pasando de un 7,2\% en el año 1980, a un 38\% en 2006, estimándose un aumento progresivo que ya para el 2012 alcanzó un 50\% (Donoso, Donoso y Arias, 2010). Se observa también mayor presencia de los quintiles de menores ingresos (I, II y III), aumentando de un $4 \%$ a un $14 \%$ el primer quintil; de un $6 \%$ a un $17 \%$ el segundo; y de un $10 \%$ a un 24\% el tercero, entre los años 1990 y 2006 (Martínez, 2011). La creciente incorporación de estudiantes de nivel socioeconómico bajo a la educación superior ha puesto sobre la mesa la discusión respecto de la concepción de igualdad de oportunidades que existe en el actual sistema de selección por méritos, ya que se ha generalizado la creencia de que esta se asocia con la equidad, sin contemplar la verdadera complejidad del fenómeno (Donoso y Schiefelbein, 2007).

De hecho, aun cuando la presencia de los sectores más desfavorecidos ha ido en aumento, esta sigue siendo muy inferior a la de otros grupos y por ello diversas universidades han implementado políticas de acción afirmativa como sistemas de ingreso alternativos a la Prueba de Selección Universitaria (PSU). Para Williamson (2007), la acción afirmativa consiste en políticas y programas que buscan abrir el acceso a oportunidades de las que diversos grupos han sido históricamente excluidos; por ende, pretenden la igualdad en el ejercicio de derechos y la equidad en el acceso a dichas oportunidades. En Chile, estas políticas se dirigen mayoritariamente a estudiantes de bajo nivel socioeconómico (en general de los tres primeros quintiles), tomando en cuenta el establecimiento educacional de procedencia. 
De acuerdo con un reporte de Unesco (2006), el éxito de los estudiantes en la educación superior estaría directamente relacionado con su desempeño en las pruebas de admisión y el tipo de establecimiento de enseñanza secundaria, elementos que a su vez se relacionan con el nivel socioeconómico de origen de los estudiantes (Díaz-Romero, 2010). En este sentido, se plantea que las instituciones de educación superior no solo deberían promover el acceso desde una auténtica lógica de equidad, sino también asumir responsabilidades en relación con la retención de los estudiantes y sus procesos educativos (Donoso y Schiefelbein, 2007). Actualmente, no existen sistemas de caracterización de los estudiantes que tomen en cuenta variables de índole afectiva (motivación, ansiedad y autoestima), cognitiva (expectativas, autoeficacia y locus de control) o social (recursos y redes de apoyo) relacionadas con la elección de carrera, por lo que no existiría un sustento empírico que oriente a las instituciones para el apoyo y seguimiento de estos procesos.

En este contexto, la elección de carrera cobra especial relevancia, ya que en muchos casos los estudiantes ingresan sin tener clara una vocación académica (Martínez, 2011). Esta falta de vocación puede desembocar en problemas de rendimiento académico, insatisfacción y deserción, con el consiguiente endeudamiento para el estudiante y su familia. Hasta el momento, las variables que podrían explicar la toma de decisión vocacional y todo lo que ella implica han sido poco estudiadas en nuestro país (Canales y De los Ríos, 2009). Considerando además que nuestro escenario educativo discrimina en favor de los sectores de mayores ingresos (Martínez, 2011), resulta relevante estudiar los procesos de elección de carrera en los sectores más vulnerables, en atención a que la pretensión actual del sistema universitario apunta a mejorar el acceso y permanencia de estos en la educación superior.

La presente investigación pretende, por tanto, explorar las creencias, significados y valoraciones respecto de la elección de carrera en estudiantes de nivel socioeconómico bajo, de dos universidades altamente selectivas de la Región Metropolitana. Se han elegido estas instituciones debido a que cuentan con programas de acción afirmativa y presentan altos niveles de exigencia académica, lo que pone a prueba los mecanismos adaptativos de los alumnos. 


\section{Elección de carrera}

El estudio de la conducta vocacional comprende el conjunto de procesos psicológicos que una persona moviliza para integrarse de manera activa al mundo profesional que le rodea (Rivas, 1989). Los elementos más estudiados han sido la madurez vocacional, los intereses vocacionales, la personalidad y su relación con la elección vocacional, la toma de decisiones, el desarrollo de carrera, la inserción profesional y las tecnologías de asesoramiento (Lobato, 2002).

A decir de Rivas (1998, 2007), los marcos conceptuales con los que se han abordado estos estudios pueden dividirse en dos categorías: una que enfatiza la dimensión individual, la responsabilidad y el control que el propio sujeto tiene en su comportamiento; y otra que destaca la dimensión socioestructural, resaltando aspectos sobre los que el sujeto no tiene ningún control, tales como demanda y oferta laboral, prestigio y movilidad social, oferta de vacantes universitarias, empleabilidad, incidencia de los avances tecnológicos, entre otros.

Sin embargo, el fenómeno vocacional se ha resistido sistemáticamente a estas miradas unidimensionales, emergiendo de forma clara su realidad multivariada:

(...) el planteamiento de lo vocacional como proceso de socialización rompe con la idea tradicional que se ha mantenido de lo que fuera la vocación como inspiración individual y en absoluto se puede sustituir por una planificación de la 'fuerza del trabajo'. Conciliar adecuadamente ambas necesidades y exigencias es quizá el reto o la utopía por la que hay que esforzarse (Rivas, 1989, p. 2).

Así, Martínez (1998) plantea una tercera categoría que denomina "enfoques interactivos", los que postulan que la conducta y el desarrollo vocacional son el resultado de la interacción de factores individuales y ambientales. Desde esta perspectiva se consideran elementos tales como las relaciones sujeto-escuela y las relaciones entorno sociodinámico-escuela, entre otros. Se agruparía en este tipo de enfoque la mayoría de los modelos evolutivos, con autores como Eli Ginzberg y Donald Super, así como los modelos cognitivoconductuales más actuales. 
En este contexto, la teoría sociocognitiva del desarrollo de carrera (SCCT por sus siglas en inglés), basada en la teoría de Bandura, ha sido articulada a partir de la década de los noventa principalmente por Robert Lent, Gail Hackett y Steven Brown, psicólogos estadounidenses que proponen que en la elección de carrera inciden factores de tipo personal (por ejemplo predisposiciones, género, raza o discapacidad), contextual (apoyo, obstáculos e influencias) y de experiencia o de agencia personal (autoeficacia, expectativas de resultado, intereses y metas) (Blanco, 2009; Cupani y Pérez, 2006; Lent y Brown, 2006; Lent, Hackett y Brown, 2004; Lent, Paixao, Da Silva y Leitao, 2010; Tracey, 2010). Esta teoría se centra en el proceso a través del cual se forman los intereses académicos y profesionales, se toman decisiones y se obtiene éxito en la escuela o en el trabajo.

En la Figura 1 puede apreciarse una representación esquemática de la SCCT:

Figura 1: Teoría sociocognitiva de desarrollo de carrera

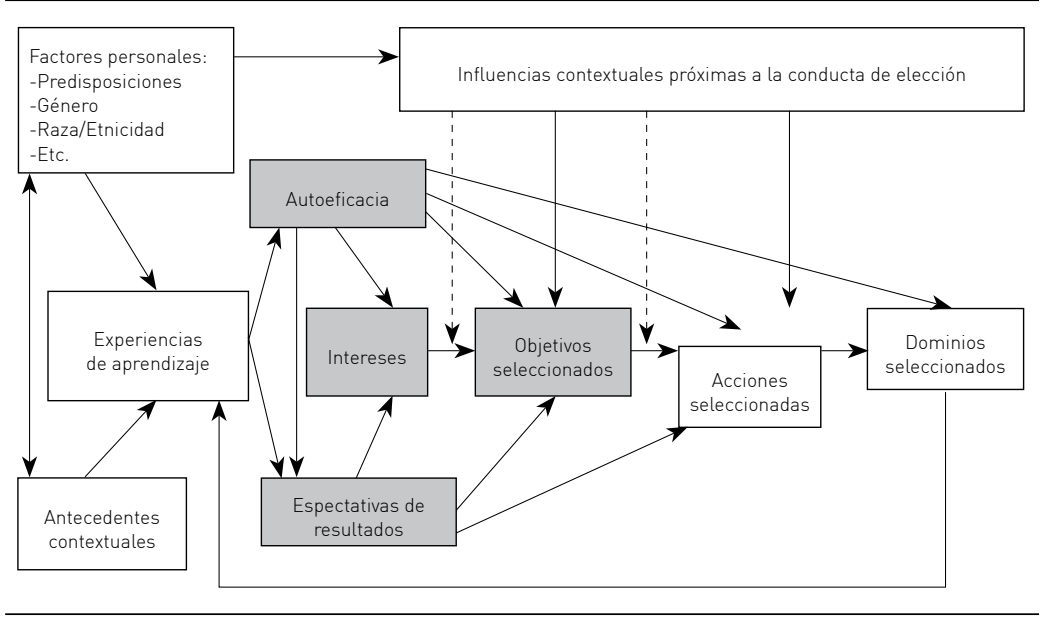

Fuente: Blanco (2009), p. 427.

En este esquema se aprecia en gris un segmento de relaciones causales que se conciben como núcleo del proceso y que considera los constructos de autoeficacia, expectativas de resultado, intereses y metas u objetivos seleccionados. La percepción de capacidad para realizar una actividad, en conjunto con la creencia de que las consecuencias serán 
favorables, determinan el desarrollo de intereses y metas. A su vez, de este núcleo se derivan ciertas acciones y logros. Se observa también que existen otras variables, de carácter personal y contextual, que afectan el proceso, y que los logros obtenidos pasan a formar parte de las experiencias de aprendizaje que lo retroalimentan (Blanco, 2009).

En el proceso de elección de carrera, la meta u objetivo sería, precisamente, escoger una de las alternativas académicas que se asocian con los intereses; las acciones orientadas a hacer la elección; y los logros derivados de la elección, por ejemplo, la aprobación o el fracaso académico (Olaz, 2003).

Este modelo teórico cuenta con abundante validación empírica y con aplicaciones prácticas en diferentes países y niveles educativos. Las investigaciones que lo han puesto a prueba han sido principalmente transversales y cuantitativas, con uso de técnicas estadísticas multivariadas, tales como modelos de ecuaciones estructurales.

Dado que en Chile no existe suficiente desarrollo de la temática específica de elección de carrera ${ }^{5}$, se justifica una primera aproximación cualitativa que permita reconocer algunos elementos que influyen en esta decisión. En este sentido, el modelo teórico de la SCCT provee de categorías que, aun cuando han sido validadas en otros contextos, facilitan el acercamiento al fenómeno y ofrecen un ordenamiento lógico de los discursos de los sujetos.

En este contexto, nos hemos planteado como objetivo de investigación identificar y describir, de manera comprensiva, aquellos factores asociados a la elección de carrera en estudiantes de nivel socioeconómico bajo que cursan primer año en universidades altamente selectivas y detectar potenciales semejanzas y diferencias entre quienes acceden a través de ingreso regular (vía PSU) y quienes lo hacen mediante programas de acción afirmativa.

5 Aunque en años recientes se aprecia un creciente interés por este tema, abordado desde diferentes perspectivas tales como motivaciones, género, equidad y consumo (Avendaño y González, 2012; González, 2007; Hernández y Paredes, 2007; León, 2010; Mizala, Hernández y Makovec, 2011; Paulus, Geoffroy, Torres y Mendoza, 2010; Rebolledo y Peña y Lillo, 2006). 


\section{Método}

La investigación se realizó desde una aproximación cualitativa, en tanto se buscó abordar comprensivamente los factores asociados a la decisión vocacional desde la perspectiva de los propios sujetos (López, Blanco, Scandroglio y Rasskin, 2010).

En cuanto a la técnica de producción de datos, se realizaron entrevistas grupales semiestructuradas, lo que permite obtener diferentes perspectivas acerca de cómo comprenden los estudiantes el proceso de elección de carrera. Cada entrevista estuvo dirigida por un moderador y un colaborador, quienes guiaron la conversación por medio de una pauta preestablecida, profundizando en las materias relevantes para el problema de investigación. Esta pauta se construyó sobre la base de información proveniente de dos fuentes: los postulados de la SCCT y entrevistas a especialistas locales, para lo cual se realizaron tres entrevistas a académicos y orientadores vocacionales.

La pauta de entrevista grupal incluyó las siguientes temáticas:

- factores generales asociados a la elección de la carrera;

- exploración de alternativas académicas;

- elección de universidad;

- fuentes de información para la elección de la carrera;

- apoyos y obstáculos en el proceso de elección de la carrera;

- experiencias asociadas a los sistemas de orientación vocacional;

- evaluación de la elección de carrera realizada; y

- factores específicos asociados a la elección de la carrera, tales como: renta, habilidades, intereses y logros esperados.

En relación con el procedimiento, se contactaron secretarías y coordinaciones de carrera, programas de acción afirmativa y unidades de bienestar de las distintas facultades y universidades, para solicitar los datos de contacto de estudiantes que cumplieran con los criterios de selección. A partir de esta información se construyó una base de datos que agrupaba a los estudiantes en función del tipo de ingreso y del área del conocimiento. La convocatoria para los participantes se realizó a inicios del año académico 2013 y consideró dos etapas: primero se les envió una invitación por correo electrónico y, en 
segundo término, se realizó una confirmación de asistencia por medio de llamado telefónico.

Es relevante señalar que las universidades en las que se realizó el estudio tuvieron una importante participación en las movilizaciones estudiantiles efectuadas durante ese año, con paralización de actividades y toma de dependencias, lo que dificultó notablemente la convocatoria a las entrevistas. Una vez finalizadas las movilizaciones, la reprogramación de actividades académicas significó una fuerte carga de trabajo para los estudiantes, lo que también redujo su participación. Esta situación fue más patente en estudiantes de Ciencias Sociales de ambas vías de ingreso, con los cuales se realizaron varias convocatorias que resultaron infructuosas, por lo que finalmente se optó por hacer entrevistas con pequeños grupos de estos estudiantes en lugar de eliminarlos de la muestra. De todos modos, se debe mencionar que con los grupos realizados se observó una saturación de los discursos de los participantes, en tanto la información fue coincidente entre las distintas entrevistas.

La primera entrevista se conformó como una prueba piloto para evaluar la adecuación de la pauta y cotejar un preanálisis de esta con el marco de referencia. A partir de esto, se hicieron las modificaciones necesarias y se construyó la pauta definitiva.

Respecto de los participantes, los criterios de selección de los estudiantes fueron los siguientes:

- pertenecer al nivel socioeconómico bajo (primeros tres quintiles);

- estudiar en una de las dos universidades en las que se realizó la investigación; y

- cursar primer año en carreras del área de la Salud, Ciencias Sociales o Ingeniería.

Estas áreas del conocimiento fueron seleccionadas por contar con un número suficiente de alumnos que ingresaron mediante programas de acción afirmativa. Los participantes se seleccionaron de acuerdo con un muestreo no probabilístico intencionado. Se realizaron siete entrevistas grupales y cada grupo fue heterogéneo respecto del género y universidad de procedencia y homogéneo en cuanto al tipo de acceso (regular o acción afirmativa) y al área del 
conocimiento. El nivel socioeconómico, entendido como un factor relevante en la elección de carrera y del éxito académico, fue similar en todos los grupos para permitir así la comparación de los discursos de los participantes de los dos tipos de ingreso.

En la Tabla 1 se presenta la composición de los grupos entrevistados.

Tabla 1: Descripción de la muestra

\begin{tabular}{|c|c|c|c|c|}
\hline$N^{0}$ & Grupo & $\begin{array}{c}\text { Número de } \\
\text { participantes }\end{array}$ & Género & Carreras \\
\hline 1 & Piloto & 9 & $\begin{array}{l}\text { Varones: } 7 \\
\text { Mujeres: } 2\end{array}$ & $\begin{array}{l}\text { Bachillerato en Ciencias y Humanidades, Geografía } \\
\text { y Administración Pública }\end{array}$ \\
\hline 2 & $\begin{array}{l}\text { PAA Ciencias } \\
\text { de la Salud }\end{array}$ & 16 & $\begin{array}{l}\text { Varones: } 8 \\
\text { Mujeres: } 8\end{array}$ & $\begin{array}{l}\text { Enfermería, Kinesiología, Fonoaudiología, Medicina, } \\
\text { Nutrición y Dietética, Obstetricia y Puericultura, } \\
\text { Tecnología Médica, Terapia Ocupacional }\end{array}$ \\
\hline 3 & $\begin{array}{l}\text { PSU Ciencias } \\
\text { de la Salud }\end{array}$ & 7 & $\begin{array}{l}\text { Varones: } 2 \\
\text { Mujeres: } 5\end{array}$ & Enfermería, Química y Farmacia, Fonoaudiología \\
\hline 4 & $\begin{array}{l}\text { PAA } \\
\text { Ingeniería }\end{array}$ & 14 & $\begin{array}{l}\text { Varones: } 7 \\
\text { Mujeres: } 7\end{array}$ & $\begin{array}{l}\text { Ingeniería Comercial, Ingeniería en Biotecnología } \\
\text { Molecular, Ingeniería en Recursos Naturales } \\
\text { Renovables, Ingeniería y Ciencias Plan Común }\end{array}$ \\
\hline 5 & $\begin{array}{l}\text { PSU } \\
\text { Ingeniería }\end{array}$ & 14 & $\begin{array}{l}\text { Varones: } 6 \\
\text { Mujeres: } 8\end{array}$ & $\begin{array}{l}\text { Ingeniería Civil en Mecánica, Ingeniería Civil en } \\
\text { Minas, Ingeniería Civil en Obras Civiles, Ingeniería } \\
\text { de Ejecución en Electricidad, Ingeniería en } \\
\text { Biotecnología, Ingeniería en Matemática, Ingeniería } \\
\text { en Microbiología, Ingeniería y Ciencias plan común. }\end{array}$ \\
\hline 6 & $\begin{array}{l}\text { PAA Ciencias } \\
\text { Sociales }\end{array}$ & 2 & Mujeres: 2 & Antropología \\
\hline 7 & \begin{tabular}{|l|} 
PSU Ciencias \\
Sociales
\end{tabular} & 3 & $\begin{array}{l}\text { Varones: } 2 \\
\text { Mujeres: } 1\end{array}$ & Antropología \\
\hline
\end{tabular}

Por último, en relación con el análisis de datos las entrevistas fueron grabadas y transcritas. A partir de estos textos se realizó un análisis de contenido categorial que consistió en dar sentido al conjunto de datos empíricos, por medio de categorías que configuran un sistema teórico que a su vez entrega coherencia a los datos extraídos (Fontes, García-Gallego, Quintanilla, Rodríguez, Rubio de Lemus y Sarriá, 2010). De esta manera, el análisis se comprende como un diálogo bidireccional entre la información producida en la interacción entre los participantes y el enfoque teórico en el que se encuadra la investigación. 
El análisis contempló cuatro etapas: preanálisis, segmentación del texto, categorización y obtención de resultados y verificación de conclusiones (Rodríguez, Gil y García, 1996). Para facilitar el procesamiento de los datos se utilizó el software Atlas Ti.

\section{Resultados}

Los discursos de los estudiantes evidencian una multiplicidad de factores asociados a la elección de carrera. Siguiendo los ejes conceptuales aportados por la SCCT, la información se ha organizado en tres amplias categorías: factores personales, contextuales y de experiencia. Los resultados se presentan en función de estas categorías, identificando, cuando resulte pertinente, las diferencias entre las dos vías de ingreso: regular y programa de acción afirmativa.

Para ejemplificar más adecuadamente los resultados se presentan citas extraídas de las entrevistas, que van acompañadas de un paréntesis que indica el grupo al que pertenece, identificando el área del conocimiento y el tipo de ingreso (PSU: ingreso regular vía PSU; PAA: ingreso vía programa de acción afirmativa).

\subsection{Factores personales}

Contemplan las características inherentes al individuo, tales como género y predisposiciones personales, que son mencionadas por los estudiantes en el relato acerca de su elección de la carrera.

\subsubsection{Género}

Considerado en la SCCT como un input personal fundamental que incide en la elección vocacional, es mencionado únicamente por los estudiantes de Ingeniería, de ambos tipos de ingreso. Los entrevistados plantean que hay ciertos campos que se asocian más a un género que al otro, como se expresa en la siguiente cita:

(...) No sé si es algo estadístico o no, a los hombres solo les sale la manía de ser con las ciencias más duras, en este caso matemática; las mujeres (...) más por la parte de la ciencia (...) por ejemplo, con biología, es por eso que en Medicina y Enfermería hay tantas mujeres (PAA-Ingeniería). 
Estos estudiantes también señalan que el género influye en la elección de una carrera específica dentro de una misma área del conocimiento:

Mecánica me convencía porque me gustan mucho las tuercas, las llaves, esas cosas, pero no me tincaba mucho porque era una carrera con muchos hombres (...). Entonces, igual la carrera que elegí fue (...) como que igual hay más hombres, pero han aumentado las mujeres (PSU-Ingeniería).

\subsubsection{Predisposiciones personales}

La SCCT plantea que las predisposiciones, características y tendencias personales debieran ser consideradas en la elección de carrera. Efectivamente, los estudiantes entrevistados, de ambos tipos de ingreso, reconocen que sus predisposiciones personales fueron un factor que incidió en su elección vocacional, buscando coherencia entre sus cualidades personales y las demandas de la carrera elegida:

(...) quería estudiar algo con salud, pero por cuestión de personalidad o carácter, como no soy muy amable, yo nunca pensé en algo que tuviese que ver con rehabilitación y tanto compromiso con los pacientes (PAA-Salud).

(...) no me gustaba así como dedicarme toda la vida a la matemática, encerrada en una oficina, porque me aburro muy fácilmente de las cosas, entonces quería algo que me mantuviera siempre entretenida (PSU-Ingeniería).

\subsection{Factores contextuales}

Corresponden a aquellos elementos socioambientales que son parte del entorno del estudiante y que facilitan o entorpecen su elección y su desarrollo de carrera. Aquí encontramos aspectos tales como los apoyos, los obstáculos y las fuentes de autoeficacia, que intervendrían en la elección académica de los estudiantes. Estos factores aparecen en los discursos de los estudiantes de las distintas áreas del conocimiento y formas de ingreso. 


\subsubsection{Apoyos}

Corresponden a diversos agentes externos que son destacados por los estudiantes por cumplir un rol de colaboración en la elección de su proyecto académico profesional. Se pueden distinguir distintos tipos de apoyos a este proceso: del entorno cercano, institucionales y económicos.

\section{a. Apoyo del entorno cercano}

Se refiere a aquellas redes sociales que soportan, alientan y refuerzan las acciones del estudiante para la elección de carrera. En este sentido, los participantes mencionan una serie de personas clave en el proceso de elección, entre ellos, su familia, profesores y amigos, los que cumplirían distintos roles, dependiendo de la vía de ingreso a la universidad.

Al respecto, la familia es uno de los principales soportes en el emprendimiento de un proyecto académico profesional, independientemente de la vía de ingreso:

(...) eso de quién nos apoyó, para mí tiene que ver mi vieja en ese sentido, porque mi vieja era como: 'no, tienes que estudiar (...) yo trabajo el doble, pero tú tenís que estudiar' (PAA-Salud).

En mi caso, mi mamá, o sea, mis papás, en general los dos, siempre me dijeron que estudiara lo que quisiera, que ellos me iban a apoyar (PSU-Ingeniería).

Estudiantes de ingreso regular relatan, además, que durante su proceso de decisión, recibieron un especial apoyo por parte de miembros de su familia que cuentan con educación superior, a través de distintas vías, por ejemplo, por medio de entrega de información acerca de la carrera:

Un tío que es bioquímico (...) como que me hizo un libro de la carrera, entonces ahí se me aclaró harto la película (PSUSalud).

O bien como un reforzamiento de la decisión: 
Mi prima hace mucho tiempo se enteró de que quería estudiar Antropología y como que dijo 'ah, qué bueno' porque ella trabaja en el tema también; se puso en contacto conmigo, me invitaba a cosas culturales, me hablaba, me motivó constantemente (PSU-Ciencias Sociales).

Y por último, como una ayuda en la postulación:

El apoyo, así como para saber, por ejemplo, cómo postular a las becas, cuándo estar pendiente de los resultados de aquí, de acá, fue mi prima que era mayor que yo, entonces ya había hecho toda la postulación, todo (PSU-Ingeniería).

Por su parte, los estudiantes que ingresan mediante programas de acción afirmativa reportan que reciben este apoyo más específico por parte de amigos que cursan estudios superiores:

Es que yo tenía un amigo que me hacía clases de matemática, entonces él estaba orientándome más bien y me mostró ciertas carreras (PAA-Ingeniería).

(...) tenía adentro como cuatro conocidos que están en cursos mayores de la carrera y eso; me orientaron harto cómo eran los primeros años (PAA-Salud).

\section{b. Apoyo institucional}

Se refiere al incentivo constante que reciben los estudiantes en su proceso de elección de carrera por parte de su colegio o liceo, el que se materializa a través de distintas actividades tales como orientación vocacional, preparación para la prueba de selección universitaria, entrega de información respecto de las carreras, organización o visita a ferias universitarias, entre otras:

El apoyo más fuerte yo creo que fue del colegio, mis compañeras, mis compañeros y los profesores (PSU-Salud).

Yo creo que lo que me influenció de forma positiva fue que siempre en el colegio nos decían (...) que si vamos a estudiar algo, teníamos que estudiar algo que nos gustara (...) siempre como que nos inculcaron en el colegio que teníamos que estudiar por vocación y no por interés económico (PAACiencias Sociales). 
Así mismo, algunos estudiantes de ingreso regular mencionan como una práctica habitual de sus establecimientos educacionales el acompañamiento en la construcción de su proyecto académico profesional:

(...) se va pasando por todas las universidades y aparte de eso todas las semanas van por lo menos dos universidades a presentar carreras y nos hacen como tres test vocacionales al año (PSU-Ingeniería).

En cambio, en los discursos de estudiantes de programas de acción afirmativa, destaca la crítica hacia sus establecimientos educacionales, debido a la inexistencia de orientación vocacional y a la falta de preparación académica para la continuación de los estudios universitarios ${ }^{6}$. Algunos estudiantes rescatan que, ante estas dificultades, determinados profesores asumen un rol activo de acompañamiento:

(...) en mi liceo, el profe de mate [matemática] (...) hizo un taller, pero no estaba dentro de su horario, o sea, él lo hizo porque él quería hacerlo (...) y todo eso porque realmente nos quería ayudar (PAA-Ingeniería).

\section{c. Apoyo económico}

Se refiere fundamentalmente a las becas a las que pueden postular los estudiantes para financiar sus carreras universitarias. Este elemento solo aparece en los discursos de estudiantes de acción afirmativa:

Yo no pago nada, o sea tengo la beca Bicentenario, tengo la beca [beca interna de la universidad] que es de mantención, tengo la beca de fotocopia, tengo beca de materiales, me dan todo. Y yo, mi familia, siempre pensaba 'entra a un técnico, que nosotros no vamos a tener para pagarte' (PAA-Ciencias Sociales).

Los estudiantes destacan que los efectos positivos de este apoyo se experimentan tanto a nivel personal como familiar:

6 Los estudiantes de liceos "emblemáticos" que ingresaron por medio de programas de acción afirmativa son la excepción, ya que reportan haber recibido apoyo en su proceso de elección vocacional en sus establecimientos educacionales. 
Igual es una repercusión para mis padres por todas esas cosas, veo ahora a las personas que tienen que pagar por lo que yo tengo gratis; (...) hay que esforzarse entonces y tomarlo como un regalo (PAA-Ingeniería).

Es relevante mencionar que, cuando la ayuda económica se asocia con las vacantes de carreras específicas, optar a estos beneficios puede implicar estudiar una carrera que no necesariamente era la primera opción del estudiante ${ }^{7}$. En estos casos, este apoyo se convierte en una encrucijada, pues representa una oportunidad única de estudiar una carrera universitaria, pero a la vez, constituye un mecanismo de presión, ya que implica renunciar a su primera preferencia.

\subsubsection{Obstáculos}

Los estudiantes, independientemente de su vía de ingreso, reconocen una serie de dificultades propias de su contexto familiar y educativo, que intervienen en su proceso de elección de carrera. Estos obstáculos son de tipo económico, del entorno cercano, institucionales y académicos.

\section{a. Obstáculos económicos}

Este factor aparece fundamentalmente en los estudiantes de acción afirmativa, quienes señalan la relevancia que su situación económica tiene en su elección de carrera:

(...) tampoco podía permitirme equivocarme y entonces, uno tiene que cumplir con lo que te ofrece la sociedad (...) decir 'sabes qué, ya, voy a dejar de estudiar, porque total tengo la plata para hacerlo'. Uno no tiene la plata para hacerlo. En cierto modo, llega un punto en el que uno tiene que aprender a que si no te gustó, obligarte a que te guste (PAA-Ingeniería).

En realidad tenía la oportunidad de estudiar la carrera de Artes y, no está como muy bien valorada, no es tan rentable y necesitaba algo rentable (PAA-Ingeniería).

Analizaremos esta tensión en el apartado de obstáculos económicos. 
Adicionalmente, como adelantamos en el apartado anterior, los estudiantes de acción afirmativa plantean que la situación económica interfiere en la elección de la carrera, particularmente cuando tienen la opción de una vacante en una carrera que no es su primera preferencia. El apoyo económico opera como una variable que modifica la elección vocacional, ya que no solo afecta al estudiante, sino también a su familia:

(...) y cuando vi que había quedado en Tecnología por XXX [menciona el cupo especial de ingreso], dije ufffff, o sea, haber trabajado y haberme pagado un preuniversitario o entrar a la segunda opción que era Tecnología Médica. Ahí pasó por mí la conciencia: esta es una carrera gratis, que es la segunda opción, que igual me gusta y aparte que era como conciencia por mi mamá, ya, me lanzo, y me matriculé y ahora me gusta lo que hago (PAA-Salud).

\section{b. Obstáculos del entorno cercano}

Refieren a la desaprobación y resistencia de la familia y del núcleo más cercano, cuando el estudiante manifiesta la preferencia por una carrera que no coincide con las expectativas familiares. Este obstáculo es mencionado por estudiantes de ambos tipos de ingreso. Las presiones van en la línea de privilegiar carreras tradicionales de mayor estatus social y económico, y rechazar aquellas con menos tradición o las del campo de las artes:

Mi papá siempre había querido que yo estudiara Medicina, Medicina, Medicina, Medicina, Medicina, Medicina (...). Cuando le dije que quería Psicología, pegó el grito en el cielo (...) 'llegaba a inflar mi pecho porque mi hija iba a ser doctora' (...) y ahora iba a ser psicóloga (...). Después de eso, cuando les dije que quería Ingeniería, ahí fue como 'yaaa, sí, sí, pero mi papá siempre me molestó con que él quería que yo estudiara Medicina (PSU-Ingeniería).

Los estudiantes se explican la resistencia de los padres sobre la base de la normal preocupación de estos por el bienestar económico de sus hijos y también por la falta de información respecto de las carreras: 
(...) la pelea entre Leyes y Teatro, entonces mi papá odiaba Teatro, porque claro me iba a morir de hambre y cuando elegí Antropología decía 'no, es que es lo mismo que Teatro' (...) pero él quería que estudiara una carrera como para subir de nivel, como ascender socialmente (PSU-Ciencias Sociales).

(...) uno que está metido maneja más información, uno sabe que estudiando Enfermería no va a ser peor que un médico, no sé, peor que un ingeniero civil, uno lo sabe y uno tiene que tratar de decirle a los papás que es así la cosa (PSU-Salud).

Aunque los estudiantes manifiestan tomar en cuenta la opinión de la familia, también destacan que no transan su elección:

Yo creo que a mi mamá se le va a quitar esa idea (...) cuando vea que estudié en verdad una carrera que me está gustando y que me aporta mucho y que tiene tanta importancia como otras carreras (PSU-Salud).

\section{c. Obstáculos institucionales}

Aquí se consideran las dificultades que los estudiantes atribuyen a sus establecimientos educacionales. Quienes ingresaron por acción afirmativa son los que plantean mayores críticas a sus colegios y liceos, tanto por la ausencia de orientación vocacional, como por la insuficiente preparación académica:

Había un orientador, pero ni él sabía de lo que se trataban las carreras (...) aquí me enteré que esa carrera es vieja, que no es tan nueva y allá ni siquiera la conocen (PAA-Salud).

En el caso mío y en el caso de otra compañera, nos miraban en menos porque decían 'ah ya, el curso se desenvuelve de esta manera, todos van a ir a instituto', entonces no, como que a las personas que quizás podrían proyectarse desde otra perspectiva no les daban ni las instancias, ni las chances para poder salir adelante (PAA-Salud).

Quería kine (Kinesiología), pero tuvimos un problema, el colegio era ratón, entonces me dio miedo que, porque igual yo tenía amigos que estaban en kine, en carreras biólogas y tenían que usar mucho microscopio y esas cosas (PAA-Ingeniería). 


\section{d. Obstáculos académicos}

Se refiere a las consecuencias que tiene para un estudiante no alcanzar el puntaje de ingreso necesario para la carrera que había elegido como primera opción. Esto es mencionado por estudiantes de ingreso regular:

Como varios acá, yo quería estudiar Medicina y por el tema del puntaje no me alcanzó, entonces tenía que buscar otra carrera de la salud, porque era el área que me gustó siempre, y no sé, de verdad no sé por qué elegí Enfermería (PSU-Salud).

El proceso de elegir el orden de la postulación más que nada es cómo le fue a uno en la PSU. Por ejemplo, en mi caso a mí me faltó tiempo para responder en matemática, entonces yo dije 'tengo que empezar a descartar opciones' (PSU-Ingeniería).

\subsubsection{Fuentes de autoeficacia}

Las fuentes de autoeficacia ejercen influencia sobre la percepción de las propias capacidades para realizar una actividad o tarea. Provienen de las personas e instituciones que ejercen persuasión social en la elección del estudiante, o bien, que actúan como modelos de aprendizaje vicario. Los estudiantes de ambos tipos de ingreso mencionan especialmente a profesores y amigos:

En el liceo yo hacía preuniversitario y el profesor que me hacía Geografía había hecho un posgrado en la universidad [...] y me habló de la carrera y a mí me interesó y empezamos a hablar mucho de eso (PAA-Ciencias Sociales).

Además que mi profesora de Física siempre me metió en la cabeza la física, me inculcó el gusto por la física (PSUIngeniería).

(...) ella salió de mi liceo, entonces nos conocíamos de ahí y cuando entró a la [nombra la universidad] me dio información y siempre me motivó. Me dijo 'sí, tú vas a quedar, y yo espero verte el otro año, y la carrera es así, tenís que hacer esto en la carrera', y como que me gustaba más, entonces como que ella igual fue como influencia (PAA-Ingeniería).

Yo creo que lo principal fue como un amigo [...] que era universitario, entonces, como yo era secundario, lo veía más 
grande, como que sabía más y él fue el que me hizo conocer la Antropología, si no no hubiera sabido y él me influenció harto yo creo (PSU-Ciencias Sociales).

\subsection{Factores de experiencia}

Son constructos que permiten describir procesos sociocognitivos complejos que intervienen en la elección de carrera y que interactúan a su vez con los factores personales y contextuales ya expuestos. Comprenden los siguientes elementos: intereses, autoeficacia, expectativas de resultados y objetivos.

\subsubsection{Intereses}

Son las preferencias hacia cierto tipo de actividades o áreas del conocimiento que intervendrían en el proceso de elección de carrera independientemente de la vía de ingreso:

Desde chiquitita, cuando todas las niñas estaban bañándose, yo estaba como mona, estaba recogiendo piedras (...) y como empecé a buscar cosas al final vi la malla de Ingeniería Civil en Minas y me llamó la atención (PSU-Ingeniería).

El optar por una carrera que implique la realización de actividades que son gratas para el estudiante le agrega un sentido de bienestar al proyecto académico profesional:

Quería algo que me conectara con la naturaleza de forma que no tenga que depender de otros factores, por ejemplo, cuando era más chico, pensaba en kine (Kinesiología) pensando que podía ser como socorrista andino, algo así, pero Geología me llamaba la atención porque podía hacer de mi trabajo algo que me gusta y en verdad igual es como solitario, pero la conexión con la naturaleza es bacán (PAA-Ingeniería).

Me gustó porque primero es común: matemático, cálculo, álgebra, y después ya como al tercer año empieza biología, división celular, entonces son cosas que a mí me gustan, entonces como que complementé las dos áreas (PSUIngeniería).

Estudiantes de ingreso regular, particularmente reconocen una trayectoria de este interés académico, que comienza en la infancia: 
Yo siempre supe, desde chico, desde pendejo, que tenía que estudiar algo relacionado con la matemática (PSU-Ingeniería).

El proceso fue superlargo, porque empezó desde que era chica siempre fui muy humanista, desde chica leía mucho, entonces siempre supe que iba pa' allá (PSU-Ciencias Sociales).

\subsubsection{Autoeficacia}

Es la valoración personal de las propias capacidades para la realización de una determinada tarea. Los estudiantes de ambas vías de ingreso describen cómo la apreciación de su desempeño ejerce una influencia en la elección de carrera, ya sea como refuerzo positivo de algunas habilidades, o bien, en el desarrollo de cierta animosidad hacia determinadas áreas.

Los estudiantes de acción afirmativa presentan una valoración negativa de su desempeño académico, que generaría autorrestricciones en su elección de carrera:

La matemática me gusta, pero no me va tan bien como yo quisiera, entonces por eso nunca fue [Campus de Ingeniería], si no que era más por el ámbito más comercial (PAAIngeniería).

En cambio, entre los estudiantes de ingreso regular se aprecia una valoración positiva de sus habilidades académicas, que es reforzada por su entorno e influye en su elección de carrera:

Me gustaba la matemática y yo también siempre tuve promedio siete en matemáticas, mis profesores siempre me decían que era buena para las matemáticas, y también era la satisfacción de resolver un problema, te da como adrenalina (risas) (PSUIngeniería).

Aunque de todos modos reportan ciertos períodos de cuestionamiento acerca de las propias capacidades:

Es como parte de esa maduración, que yo siento que habría sido imposible adquirirla antes, como el hecho de pensar como 'nadie entra sabiendo todo' y que en verdad tú no compites con los demás, compites contigo (PSU-Salud). 
Dentro del apoyo, como el de mí mismo, reforzando mi idea de que yo era capaz de lograr lo que me proponía, porque como necesitaba reforzarme esa idea de mí mismo, de mantener firme mi autoestima (PSU-Ciencias Sociales).

Un discurso transversal de los estudiantes, ya realizada la elección es la confianza en sus capacidades para alcanzar la meta, en este caso, obtener la carrera que se pretende estudiar:

No se tiene que pensar 'yo no puedo, no tengo las capacidades', o 'no, yo no puedo porque no, la gente rica estudia eso', no, o sea, si uno hace su máximo esfuerzo, uno puede, yo creo que ahí uno tiene que convencerse de que puede (PAA-Ciencias Sociales).

(...) conocí los rojos y todo, pero no pienso cambiarme de carrera, me gusta y aunque me cueste, la voy a sacar sí o sí (PSU-Ingeniería).

\subsubsection{Expectativas de resultados}

Son aquellas consecuencias esperadas de la elección de carrera y del propio desempeño académico y profesional. Los estudiantes de ambas formas de ingreso identifican dos tipos de expectativas: de estatus social y económico y de contribución a la sociedad.

\section{a. Expectativas de estatus social y económico}

Aquí se presentan ejemplos como los siguientes:

Los ingenieros, en general los comerciales, ganan harto y eso para mí es importante, sé que es supermaterialista y todo eso, pero a mí me importa, si los ingenieros comerciales no ganaran nada, seguramente no lo voy a estudiar, entonces el posicionamiento y el dinero que pueda obtener después es importante para lo que uno quiera hacer, tanto bien o mal, para mí igual fue importante eso (PAA-Ingeniería).

(...) la carrera me gustó porque tiene buena proyección laboral, es una de las mejores con proyección laboral (PSUIngeniería). 
Estas expectativas de renta estarían asociadas también a la potencial contribución al sustento familiar:

Mi mamá es temporera y va a llegar un momento en que mi hermana chica va a crecer, se va a ir y va a quedar sola y a nosotros nos va a quedar cuidarla y eso po', y yo veo más que nada también por mi hermana y por eso de estudiar algo que me guste, si igual me gusta y que me dé plata (PAA-Salud).

Mi sueño, antes de que mis papás y mi tata fallezcan, es que, no sé, darles un viaje por toda Europa, por todo el mundo, es como ni siquiera comprarme una casa yo o un auto, lo que sea, sino que darles a ellos todo lo que me dieron a mí y por ejemplo, por eso igual estudio todos los días o hasta tarde (PSU-Ingeniería).

En los estudiantes de ingreso regular cobra mayor fuerza la consideración de la universidad en la elección de la carrera, ya que la casa de estudios se asocia con el prestigio social:

Te inculcan como desde chico que la [nombra la universidad] es lo mejor, te venden la pomá que la [nombra la universidad] no sé qué, que tenís que estudiar en la [nombra la universidad], que te hace ser mejor persona, entonces yo creo que eso también es como una presión a que uno tiene que quedar en la [nombra la universidad] (PSU-Salud).

También se relaciona con mejores expectativas laborales:

Es como competitiva porque hay pocos antropólogos y bajo ese prejuicio es como que se estudia en la [nombra la universidad], para que te vaya mejor en el tema laboral, siempre con eso que el currículo vale mucho, la universidad es como un respaldo (PSU-Ciencias Sociales).

Y por último con la excelencia académica:

Postulé en primera opción en la [nombra la universidad] por el tema de que la carrera que yo estudio es como, según lo que investigué, la [nombra la universidad] es la que mejor la imparte (PSU-Ingeniería). 
b. Expectativas de contribución a la sociedad Aquí se evidencian comentarios como los siguientes:

(...) soy de las personas que va a hospitales públicos, que no tenemos los ingresos como para ir a una clínica privada (...) entonces uno ve el sistema, uno lo vive y uno dice 'yo quiero realizar un cambio en esto', entonces la única forma de poder realizar un cambio es ejerciendo en ese ámbito, entonces: ¿cómo puedo cambiar ese lugar? Ejerciendo, estudiar una carrera que sea en ese ámbito y cuando tenga mi título en mano, trabajar en el área salud (PSU-Salud).

Este elemento puede ir en contraposición a las expectativas de renta, especialmente cuando se elige una carrera cuyo campo laboral está asociado con el ámbito público. En estos casos, en la decisión de los estudiantes prima la contribución social que podrán hacer con su desempeño profesional:

Yo sé que con esta carrera no me voy a hacer millonario ni mucho menos, pero me gratifica saber que yo estoy trabajando para las demás personas y que ellos se complacen al ver que en vez de hacer una cola de tres horas, la pueden hacer en una (PAA-Ciencias Sociales).

\subsubsection{Objetivos}

Finalmente entre los factores de experiencia está el objetivo, que corresponde al logro de elegir una carrera, lo que es considerado en sí mismo una meta. En este análisis se contemplan las acciones seleccionadas para alcanzar la meta y los significados asociados a la elección.

\section{a. Acciones seleccionadas}

En este caso los estudiantes describen diversas acciones durante el proceso de preparación de su proyecto académico profesional, específicamente para tomar la decisión vocacional. Estas estrategias son: la búsqueda de información por Internet, la entrevista con profesionales y otros actores y la participación en actividades específicas. 
Uno de los primeros pasos, previamente a la decisión vocacional, es la exploración en Internet:

Cuando llegué a $3^{\circ}$ o $4^{\circ}$ ya empecé a investigar en Internet las páginas de las universidades, las carreras, vi las mallas (PSU-Ingeniería).

En este mismo sentido también buscan opiniones de profesionales o estudiantes de las carreras de interés, especialmente por la falta de conocimiento que reconocen acerca de estas:

Yo pesqué el teléfono, marqué el $007^{8}$ y dije 'necesito un fonoaudiólogo', no tenía idea, no cachaba, mi amiga me dijo 'tiene unas áreas clínicas', pero nunca vi un fonoaudiólogo, nunca lo vi en el hospital, nunca escuché. Y el caballero me contestó y me dijo que si necesitaba una hora y le dije: 'no, necesito hablar con usted para que me explique qué onda su carrera' y me dijo 'yo soy de los primeros egresados de la [nombra la universidad] y me dijo, 'no, la carrera es esto, esto y esto' y me con tó que ahora estaba la innovación en la escuela y que la malla iba innovándose y ahí me convencí (PAA-Salud).

Me contacté con universitarios de diferentes carreras para hablar con ellos, porque una cosa es la pomada que te vende la universidad, porque al final te están ofreciendo un producto, y otra cosa es lo que dice la persona real, entonces ya, y me puse a conversar con ellos (PSU-Ingeniería).

Algunos estudiantes reportan haber participado en actividades específicas asociadas al área de la carrera, lo que les permite un acercamiento a su praxis:

Yo tuve la posibilidad de estar en una escuela de verano enfocada en lo que era la Geografía, Diseño y Arquitectura, podíamos vivir un poco de lo que se trataba, yo creo que la práctica ayuda mucho en la decisión (PAA-Ciencias Sociales).

Yo lo que hice fue aparte de ver en Internet, fue meterme en unos cursos que están (...) unos cursos introductorios que estaban haciendo en la [nombra la universidad] (...) tenían un curso de diez días para participar de Antropología,

Servicio de información telefónica móvil. 
explicaban qué era la carrera, cuáles eran tus oportunidades laborales, de hecho como que nos sacaron a dar una vuelta (PSU-Ciencias Sociales).

\section{b. Significados asociados a la elección de carrera}

Los discursos de los estudiantes expresan que la elección de carrera tiene diversos significados para ellos. Por una parte, los estudiantes de ingreso regular la describen como un hito que marca su vida y la asumen como una decisión personal, en la que desplazan a otros actores relevantes como la familia y las amistades:

Dije por primera vez 'voy a tomar la decisión más importante que he tomado en mi vida y voy a decidir por mí'. Entonces al final tomé amigos, familia y los dejé a un lado, me voy al computador y busco universidades, lo único que quería (PSU-Ingeniería).

En cambio, los estudiantes de acción afirmativa describen el ingreso a la universidad como un hito que es relevante no solo en el ámbito personal, sino también para toda la familia:

Soy como la primera generación de mi familia que entró a la universidad, entonces a lo mejor ni siquiera es por uno, pero es después mi mamá, porque yo soy de familia separada (PAA-Salud).

Otro elemento interesante de destacar en relación con los significados es el proceso de encantamiento con la carrera estudiada, en los casos en los que esta no era la primera preferencia:

Por lo menos yo estoy feliz, bueno igual no era lo que yo quería estudiar, yo quería estudiar Medicina, pero cuando me llegó la hora de que tenía visitas de observación y todas esas cosas (...) creo que ahí ya me di cuenta que era la elección correcta (PAA-Salud).

Finalmente, y como una síntesis de los resultados ya presentados, las Figuras 2 y 3 presentan la red de relaciones entre los elementos identificados como relevantes en el proceso de elección de carrera por los estudiantes de ambos tipos de ingreso. 
Figura 2: Elementos identificados como relevantes en su proceso de elección de carrera por estudiantes de ingreso regular

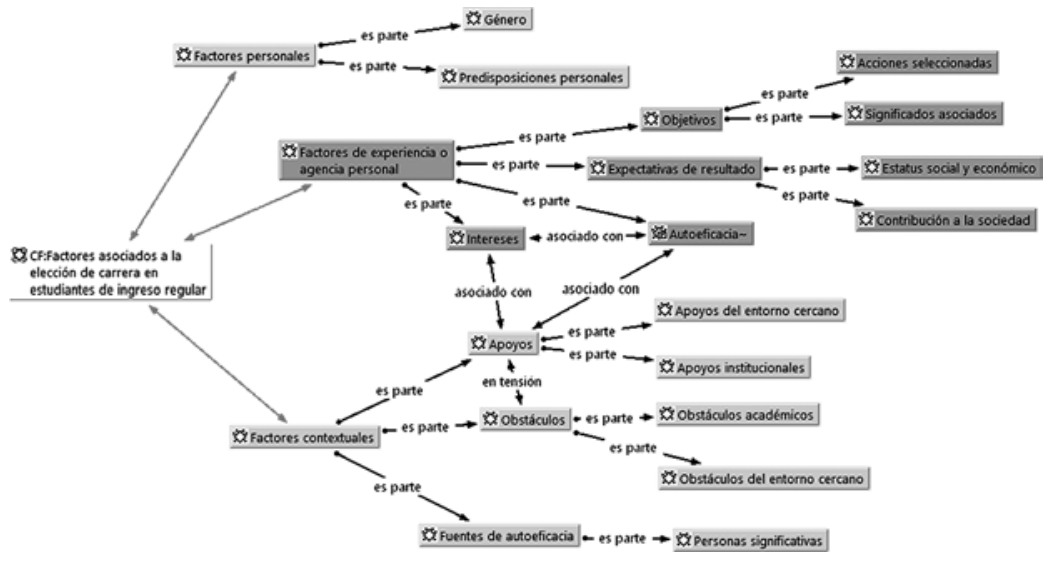

Fuente: Elaboración propia.

Figura 3: Elementos identificados como relevantes en su proceso de elección de carrera por estudiantes de acción afirmativa

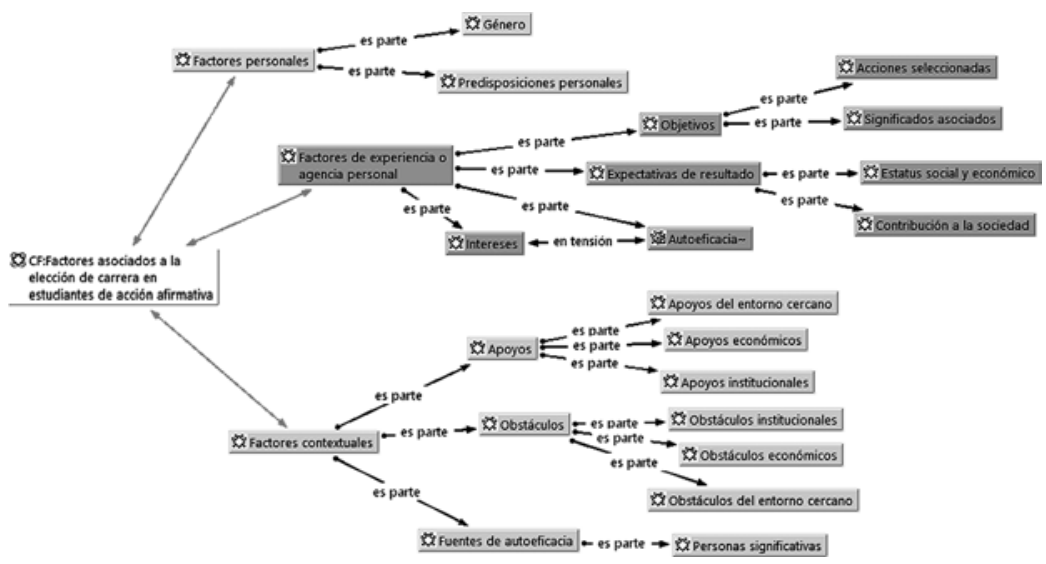

Fuente: Elaboración propia.

\section{Conclusiones}

Siguiendo los ejes conceptuales aportados por la SCCT, todos los elementos identificados por los estudiantes como relevantes en su 
proceso de decisión vocacional han sido agrupados en función de tres categorías: factores personales, factores contextuales y factores de experiencia.

En relación con los factores personales, en los discursos de los estudiantes se recogen dos tipos de factores de este tipo que incidirían en su elección de carrera: las predisposiciones personales, que son señaladas por estudiantes de ambos tipos de ingreso y de distintas áreas del conocimiento; y el género, que solo es mencionado por estudiantes de Ingeniería, de los dos tipos de ingreso. Estos factores personales no son considerados por los estudiantes de forma aislada en su elección de carrera, sino que se reflejan en la búsqueda de confluencia entre las propias características y las de la carrera a estudiar. De este modo, este factor opera sobre la base de cierto conocimiento acerca del campo laboral, o al menos, de ciertas ideas preconcebidas respecto de este, dejando en evidencia la relevancia que tiene, para una adecuada elección, el que los estudiantes puedan contar con información oportuna y fidedigna respecto de las distintas carreras, que vaya más allá de las creencias propias del sentido común.

Acerca de los factores contextuales, estos se agrupan en apoyos, obstáculos y fuentes de autoeficacia. La familia se reconoce como un soporte fundamental, independientemente de la vía de ingreso de los estudiantes, pero en el caso de quienes ingresan vía PSU, los familiares con estudios superiores parecen tener un rol más activo en la decisión propiamente tal, ya que tienen la posibilidad de entregar al estudiante información acerca de las carreras y aconsejarlos en el proceso de postulación a la universidad. En la situación de los estudiantes de acción afirmativa, este papel lo asumen amigos que cursan estudios superiores.

En el marco del apoyo institucional se observan diferencias según el tipo de ingreso. Así, mientras los estudiantes de ingreso regular reconocen haber recibido apoyo en sus colegios o liceos para la elección y preparación universitaria, los estudiantes de acción afirmativa describen fuertes carencias en sus establecimientos educacionales. Ante este importante déficit, algunos participantes rescatan el rol de profesores que, por iniciativa propia, intentan compensar estas carencias. 
El apoyo económico, como factor que facilita la decisión vocacional, destaca en los discursos de los estudiantes de acción afirmativa, quienes describen estos programas especiales de ingreso como una oportunidad invaluable para desarrollar un proyecto académico profesional.

En cuanto a los obstáculos, los estudiantes de ambos tipos de ingreso reportan algunas resistencias por parte de su entorno cercano cuando se pretende estudiar carreras no tradicionales, de menor estatus social o económico. Aquí resulta interesante verificar cómo los estudiantes se explican en parte esas resistencias, debido a la falta de información disponible acerca de las carreras menos tradicionales.

Entre los estudiantes de acción afirmativa se relevan los obstáculos institucionales, tales como la falta de orientación vocacional, la subvaloración del potencial de los estudiantes y las falencias en su formación académica, elementos que afectan tanto la toma de decisión vocacional, como el posterior rendimiento académico en la universidad.

Otro tipo de obstáculo que destaca en el discurso de estudiantes de acción afirmativa es el de índole económica. En este sentido es importante destacar que, aunque todos los entrevistados son de nivel socioeconómico bajo, las dificultades económicas parecen tener mayor influencia en la elección de carrera de estudiantes que han ingresado a la universidad por la vía de programas de acción afirmativa. En este sentido, este tipo de iniciativas, valoradas tan positivamente por los estudiantes y sus familias, se experimentan a la vez como una restricción a las posibilidades de elección, cuando se asocian a cupos en carreras que no necesariamente coinciden con la primera preferencia del estudiante. Sin duda, esta es una tensión que requiere ser estudiada en futuras investigaciones.

Los estudiantes de ingreso regular describen, además, obstáculos de tipo académico, que se refieren a no alcanzar el puntaje PSU necesario para ingresar a la carrera de su primera preferencia, lo que los lleva a elegir otra dentro de la misma área del conocimiento, pero de menor estatus o que no los satisface plenamente. Por tratarse 
de estudiantes de universidades altamente selectivas, en muchas ocasiones el puntaje obtenido les habría permitido estudiar la carrera deseada en otra universidad; sin embargo, ellos reportan haber privilegiado el prestigio de la institución por encima de la carrera que deseaban. Esto debiera ser profundizado en futuros estudios, pues es posible que priorizar el prestigio de la institución, por sobre la carrera esté asociado al nivel socioeconómico de los estudiantes entrevistados, quienes no cuentan con redes sociales y/o familiares que les aseguren una adecuada inserción laboral al egresar de la universidad. En este sentido, tener un título profesional de una universidad de alto prestigio podría facilitarles ese proceso.

En cuanto a las fuentes de autoeficacia, llama la atención que los estudiantes reporten dos agentes de influencia: profesores y amigos; mientras que la familia aparece ausente de este rol. Considerando que la familia es reconocida por los entrevistados como una fuente importante de apoyo, podemos suponer que su escasa influencia sobre la percepción del estudiante acerca de sus propias capacidades no se deba ni a un escaso interés por parte de la familia, ni a falta de confianza de esta en el potencial del estudiante. Una hipótesis a poner a prueba en futuras investigaciones es que, por tratarse de estudiantes de nivel socioeconómico bajo, en su mayoría primera generación de universitarios, la familia nuclear no cuenta con la formación necesaria para jugar un rol activo en la preparación académica del estudiante, desde donde poder observar y reforzar sus capacidades.

Ahora, entre los factores de experiencia, los intereses y preferencias de los estudiantes por ciertas actividades y campos del conocimiento son elementos que destacan como relevantes en su elección de carrera. Se observan diferencias según el tipo de ingreso: quienes acceden vía PSU relatan con mayor frecuencia una cierta trayectoria en el desarrollo de la elección de carrera que se construye desde la infancia. En cambio, en estudiantes de acción afirmativa aparece más comúnmente el relato de que la elección se consolida recién al constatar que podían acceder a la universidad a través de estos programas especiales. Podemos hipotetizar que lo tardío de la decisión vocacional en estos estudiantes se asocia a la falta de expectativas respecto de la posibilidad de ingresar a la educación 
superior, lo que implicaría que el alumno no se vea en la necesidad, o bien, no se permita la posibilidad de realizar una elección temprana. Teniendo en cuenta la relevancia que la elección de carrera tiene en el desempeño académico del estudiante, este elemento aparece como un tema para ser trabajado por parte de las instituciones educativas, con el objetivo de que los programas de acción afirmativa faciliten no solo el acceso a estudiantes de sectores vulnerables, sino también su adecuada adaptación y retención en el sistema.

La autoeficacia es otro de los elementos referidos por los estudiantes como relevante en su proceso de elección de carrera y operaría de distinto modo según el tipo de ingreso. Así por ejemplo los estudiantes de acción afirmativa presentan una negativa valoración de su desempeño académico, lo que restringe su elección de ciertas carreras; en cambio los estudiantes de ingreso regular valoran positivamente sus cualidades académicas y tienen mayor confianza, por tanto, en su futuro desempeño.

En el marco de las expectativas de resultados, los discursos de los estudiantes indican que, independientemente de la vía de ingreso, son dos los tipos de expectativas que influyen en su elección de carrera: el estatus social y económico y la posibilidad de realizar una contribución a la sociedad.

En cuanto al objetivo de elegir una carrera, los estudiantes de ambos tipos de ingreso realizan acciones similares para llegar a tomar su decisión. Sin embargo, los estudiantes de ingreso regular reportan mayor sistematicidad en la búsqueda de información acerca de los campos y carreras de interés. Respecto de los significados asociados a la elección de carrera, también se observan diferencias en función del tipo de ingreso. Los estudiantes de ingreso regular la describen como un hito fundamentalmente individual, mientras que estudiantes de acción afirmativa la viven como un proceso que involucra también a toda su familia.

Entre los significados que los estudiantes otorgan a la elección, resulta interesante observar que cuando los jóvenes optan por una carrera que no era su primera preferencia, se produce un proceso de 
ajuste, durante el cual el estudiante flexibiliza sus argumentos y logra reconocer características positivas de la carrera estudiada, que son coherentes con sus preferencias. Podemos hipotetizar que este proceso corresponde a lo que Festinger (1975) identifica como estrategias para eliminar o reducir la disonancia cognitiva, lo que abre nuevas interrogantes para futuros estudios.

En síntesis, esta investigación ha permitido identificar elementos que, según sus propios discursos, habrían incidido en el proceso de elección de carrera de estudiantes chilenos de nivel socioeconómico bajo, que cursan primer año en universidades altamente selectivas de la Región Metropolitana y que han ingresado a través de PSU, o bien, por medio de programas de acción afirmativa.

Se abren distintas alternativas para el desarrollo de nuevas investigaciones, que pueden ir en la línea de replicar el trabajo con estudiantes del mismo nivel socioeconómico de universidades no selectivas, o bien, en estudiantes de las mismas universidades selectivas, pero de nivel socioeconómico medio y alto, para estudiar las coincidencias y diferencias en los factores que estarían implicados en una decisión tan relevante para el proyecto académico profesional de un joven, como es la elección de carrera.

Otra línea de desarrollo debiera considerar el género y el territorio, incluyendo universidades regionales, de manera de establecer nuevas comparaciones que nutran la comprensión del fenómeno. También parece necesario continuar estudiando las particularidades de este proceso en estudiantes que ingresan vía programas de acción afirmativa, de modo de poder identificar aquellos factores asociados a un mayor riesgo de deserción, con el fin de que las instituciones educativas puedan implementar políticas destinadas a favorecer la adecuada inserción y el desempeño exitoso de estos estudiantes.

Finalmente, podemos concluir que una contribución del presente trabajo es la constatación de que las categorías desprendidas desde la teoría sociocognitiva de carrera han resultado de utilidad para ordenar y comprender los diversos elementos que los estudiantes entrevistados han identificado como relevantes en su proceso 
de elección de carrera. Esto indicaría que, a pesar de haber sido desarrollado en otro contexto, este modelo teórico puede ser un aporte a la comprensión del proceso de elección de carrera en un contexto educativo tan particular como el chileno.

\section{Referencias}

Avendaño, C. y González, R. (2012). Motivos para ingresar a las carreras de Pedagogía de los estudiantes de primer año de la Universidad de Concepción. Estudios Pedagógicos, 38(2), 21-33.

Blanco, A. (2009). El modelo cognitivo social del desarrollo de la carrera: revisión de más de una década de investigación empírica. Revista de Educación, 35, 423-445.

Canales, A. y De los Ríos, D. (2009). Entendiendo la permanencia de estudiantes vulnerables en el sistema universitario chileno. Revista Calidad en la Educación, 30, 1-38. Recuperado el día 30 de abril de 2011 desde http://www.cned.cl/public/secciones/SeccionRevistaCalidad/ doc/52/CSE_resumen 817.pdf

Cupani, M. y Pérez, E. (2006). Metas de elección de carrera: contribución de los intereses vocacionales, la autoeficacia y los rasgos de personalidad. Interdisciplinaria, 23(1), 81-100.

Díaz-Romero, P. (2010). Universidades de calidad: universidades inclusivas. Fundación Equitas. Recuperado el día 25 de mayo de 2012 desde http:// www.fundacionequitas.org/descargas/Pamela_Diaz-Romero_WS_III.pdf

Donoso, S., Donoso, G. y Arias, O. (2010). Iniciativas de retención de estudiantes en educación superior. Revista Calidad en la Educación, $33,15-61$.

Donoso, S. y Schiefelbein, E. (2007). Análisis de los modelos explicativos de retención de estudiantes en la universidad: una visión desde la desigualdad social. Estudios Pedagógicos, 33(1), 7-27.

Festinger, L. (1975). Teoría de la disonancia cognitiva. Madrid: Instituto de Estudios Políticos. (Obra original publicada en 1957).

Fontes, S., García-Gallego, C., Quintanilla, L., Rodríguez, R., Rubio de Lemus, P. y Sarriá, E. (2010). Fundamentos de investigación en Psicología. Madrid: Universidad Nacional de Educación a Distancia.

González, B. (2007). Desigualdad social, género y educación superior en Chile. ¿Elección educativa adscrita a la función social materna o independencia de género? CIPS Chile. Recuperado el día 5 de abril de 2012 desde http:// www.cips.cl/contenidos/soc/SOC-02.pdf 
Hernández, L. y Paredes, R. (2007). Restricciones económicas en la decisión de continuar estudios superiores técnicos o profesionales. Revista Calidad en la Educación, 27, 238-261.

Lent, R. \& Brown, S. (2006). On conceptualizing and assessing social cognitive constructs in career. Journal of Career Assessment, 14(1), 12-35.

Lent, R., Hackett, G. \& Brown, S. (1994). Toward a unifying social cognitive theory of career and academic interest, choice and performance. Journal of Vocational Behavior, 45, 79-122.

Lent, R., Hackett, G. y Brown, S. (2004). Una perspectiva social cognitiva de la transición entre la escuela y el trabajo. Evaluar, 4, 1-22.

Lent, R., Paixao, M., Da Silva, J. \& Leitao, L. (2010). Predicting occupational interests and choice aspirations in Portuguese high school students: a test of social cognitive career theory. Journal of Vocational Behavior, $76,244-251$.

León, M. (2010). Factores que condicionan la elección de una carrera universitaria por parte de los alumnos de último año de secundaria en Chile. Tesis de Doctorado en Marketing, Operaciones y Finanzas, Universitat Ramon Llull. Recuperado el día 30 de julio de 2012 desde http://www.tdx.cat/ bitstream/handle/10803/9183/LEON_Tesis_Doctoral.pdf?sequence=1

Lobato, C. (2002). Psicología y asesoramiento vocacional. Revista de Psicodidáctica, 13. Recuperado el día 8 de junio de 2012 desde http:// www.doredin.mec.es/documentos/ 01520073000061.pdf

López, J., Blanco, F., Scandroglio, B. y Rasskin, I. (2010). Una aproximación a las prácticas cualitativas en Psicología desde una perspectiva integradora. Papeles del Psicólogo, 31(1), 131-142. Recuperado el día 4 de enero de 2011 desde: http://www.papelesdelpsicologo.es/ pdf/1803.pdf

Martínez, M. (1998). Orientación vocacional y profesional. Papeles del Psicólogo, 71. Recuperado el día 8 de julio de 2012 desde http://www. papelesdelpsicologo.es/vernumero.asp?id=794

Martínez, M. (2011). Programas inclusivos: el reto de la equidad en el acceso a la educación universitaria en Chile. Tinkazos, 14(30), 145-165.

Mizala, A., Hernández, T. y Makovec, M. (2011). Determinantes de la elección y deserción en la carrera de Pedagogía. Educación 2020. Recuperado el día 25 de julio de 2013 desde http://www. educacion2020.cl/index.php?option=com_docman\&task=doc_ download\&gid=210\&Itemid=55

Olaz, F. (2003). Modelo social cognitivo del desarrollo de carrera. Evaluar, $3,15-34$. 
Paulus, N., Geoffroy, E., Torres, R. y Mendoza, M. (2010). Descripción de criterios presentes en la selección de instituciones y carreras de educación superior y su segmentación por género. Cedus. Recuperado el día 25 de julio de 2012 desde http://www.cned.cl/public/Secciones/ SeccionInvestigacion/investigacion_estudios_y_documentos_de_ genero_pdf.aspx?strArt=Descripci\%F3n\%20de \%20 criterios\%20 presentes\%20en\%20la\%20selecci\%F3n\%20de\%20instituciones\%20 y\%20carreras\%20de\%20educaci\%F3n\%20superior, $\% 20$ y 20 su $\% 20$ segmentaci\%F3n\%20por\%20g\%E9nero.\&idArticulo=927

Rebolledo, L. y Peña y Lillo, M. (2006). Elección de carreras universitarias y género en el caso de Trabajo Social e Ingeniería. Tesis de Magíster en Estudios de Género y Cultura en América Latina, mención en Ciencias Sociales, Universidad de Chile. Santiago: Universidad de Chile. Recuperado el día 4 de julio de 2012 desde http://www.cybertesis.cl/ tesis/uchile/2006/arredondo_m/html/index-frames.html

Rivas, F. (1989). El asesoramiento vocacional como relación de ayuda técnica. Reflexión teórica y resultados de investigación. Papeles del Psicólogo, 39 y 40. Recuperado el día 8 de mayo de 2011 desde http://www. papelesdelpsicologo.es/vernumero. .asp?id $=400$

Rivas, F. (1998). Psicología vocacional: enfoques del asesoramiento. Madrid: Morata.

Rivas, F. (2007). ¿Conducta y asesoramiento vocacional en el mundo de hoy? Revista Electrónica de Investigación Psicoeducativa, 5(1), 5-14.

Rodríguez, G., Gil, J. y García, E. (1996). Metodología de la investigación cualitativa. México: Ediciones Aljibe.

Tracey, T. (2010). Relation of interest and self-efficacy occupational congruence and career choice certainty. Journal of Vocational Behavior, $76,441-447$.

Unesco. (2006). Compendio mundial de educación 2006. Comparación de las estadísticas de educación en el mundo. Montreal: Unesco. Recuperado el día 28 de julio de 2011 desde http://www.uis.unesco.org/Library/ Documents/ged06_es.pdf

Williamson, G. (2007). Acción afirmativa en educación superior: dilemas y decisiones en tiempos multiculturales de globalización. Ciencias Sociales Online, 4(1), 77-101. Recuperado el día 8 de agosto de 2012 desde http://www.uvm.cl/csonline/2007_l/pdf/accion.pdf

Recibido: 02/01/2014

Aceptado: 06/06/2014 